\title{
Uso de recursos $y$ rendimiento en las actividades evaluación: análisis y comparación de resultados en el Grado en Ingeniería de Tecnologías Industriales ${ }^{1}$
}

\section{Begoña Peña ${ }^{a}$}

a Universidad de Zaragoza - Departamento de Ingeniería Mecánica (España) e-mail: bpp@unizar.es.

\begin{abstract}
Virtual Learning Environments such as Moodle register a large amount of information about student activity that is normally hidden for both the teacher and the student. This paper presents a simple analysis of this information from the data processing in a spreadsheet, without the help of tools that automate processing. In order to evaluate its usefulness, a comparison of the activity and the learning results is here presented for the last three courses on the subject Engineering Thermodynamics and Fundamentals of Heat Transmission of the Degree in Engineering of Industrial Technologies at the University of Zaragoza. The results show the patterns of the different groups of students, allowing to detect deviations and to predict in some extent the success in the final exams.
\end{abstract}

Keywords: Moodle, Learning Analytics, High Education Innovation, Engineering.

\begin{abstract}
Resumen
Las plataformas digitales educativas como Moodle recogen una gran cantidad de información sobre la actividad del estudiante que normalmente queda oculta tanto para el profesor como para el alumno. En este trabajo se presenta un análisis sencillo de dicha información a partir del tratamiento de datos en una hoja de cálculo, sin ayuda de herramientas que automaticen el procesamiento. Con el fin de evaluar su utilidad, se ha realizado la comparativa de la actividad y de los resultados de aprendizaje de los tres últimos cursos para la asignatura de Termodinámica Técnica y Fundamentos de Transmisión de Calor del Grado en Ingeniería de Tecnologías Industriales de la Universidad de Zaragoza. Los resultados muestran los patrones de los diferentes grupos de estudiantes, permitiendo detectar desviaciones e incluso predecir en cierta medida el éxito en los exámenes finales.
\end{abstract}

Palabras clave: Moodle, Learning Analytics, Innovación Universitaria, Ingeniería.

\footnotetext{
1 Este trabajo se ha desarrollado dentro del proyecto PIIDUZ_17_299 de innovación docente (Programa de Proyectos de Innovación Docente para Grupos de Profesores del Vicerrectorado de Política Académica de la Universidad de Zaragoza).
} 


\section{Introducción}

Las plataformas educativas se han convertido para la Universidad en un entorno habitual de comunicación entre alumnos y profesores, en donde se encuentran disponibles los recursos de las asignaturas. Además, gracias a las nuevas funcionalidades, como foros, wikis o cuestionarios, se utlizan cada vez más como herramienta de aprendizaje y de evaluación. Esto hace que cada usuario deje una huella digital de sus actividades que se puede analizar y procesar para establecer patrones de conducta o de aprendizaje, informar al estudiante y al profesor de los progresos y predecir el éxito final (Boyer, 2016). Todo esto ha abierto un nuevo campo de investigación, las analíticas del aprendizaje (Learning Analytics, LA) cuyo potencial solo se empieza a vislumbrar.

Reino Unido es pionero en la implementación de estas herramientas a nivel nacional (Sclater, 2016), pero no es el único país que está convencido del potencial de LA. La Comisión Europea está trabajando en este sentido para desarrollar guías y medios que faciliten la adaptación de LA en la Universidad Europea con el fin de asegurar la calidad (Ferguson, 2016). En otros países como Estados Unidos o Australia, también se lleva años desarrollando herramientas y modelos predictivos basados en LA que ayuden a mejorar los resultados de aprendizaje (Wanli, 2015).

En este sentido, parece demostrado que puede utilizarse como herramienta a nivel institucional para asegurar y mejorar la calidad, para monitorizar el seguimiento y el progreso en el aprendizaje de una asignatura, para reducir las tasas de abandono identificando a tiempo los casos de riesgo y como precursor del, tan perseguido, aprendizaje adaptativo o personalizado.

El presente trabajo se ha desarrollado siguiendo esta prometedora línea de investigación educativa con el fin de utilizar el potencial de LA para mejorar los resultados de aprendizaje en el Grado en Ingeniería de Tecnologías Industriales de la Universidad de Zaragoza. Concretamente, en esta ponencia, se presentan los datos recogidos de la plataforma Moodle en la asignatura de Termodinámica Técnica y Fundamentos de Transmisión de Calor para los tres últimos cursos académicos. Puesto que se han ido incorporando materiales y actividades durante este tiempo, la comparativa no puede ser completa y se realizará en base a aquellas actividades que son comunes al menos a dos cursos académicos.

\section{Objetivos}

Este trabajo ha surgido de las diferencias encontradas en las calificaciones finales para una misma asignatura de dos cursos académicos consecutivos en los que se ha seguido la misma metodología de aprendizaje y evaluación con el mismo profesor. Este hecho ha planteado la necesidad de obtener más información sobre el proceso de aprendizaje para encontrar los factores que más influyen en los resultados de aprendizaje. 
Los objetivos específicos de este trabajo son:

- Recopilar datos almacenados en cada curso en la plataforma Moodle.

- Procesar los datos para obtener indicadores promediados de dedicación y esfuerzo del estudiante en la asignatura.

- Comparar dichos indicadores para los diferentes cursos académicos.

- Obtener patrones y seleccionar los parámetros que pueden ser útiles para predecir el éxito en la asignatura.

\section{Desarrollo de la innovación}

El presente estudio se ha llevado a cabo con alumnos del Grado en Ingeniería de Tecnologías Industriales de la Universidad de Zaragoza, concretamente en la asignatura "Termodinámica Técnica y Fundamentos de Transmisión de Calor", que se imparte durante el primer cuatrimestre del segundo curso. La asignatura cuenta con 6 ECTS, equivalentes a 150 horas de trabajo, correspondientes a 45 horas de clases presenciales de teoría y resolución de problemas, 15 horas de prácticas de laboratorio y 90 horas de trabajo personal del alumno (estudio individual, resolución de problemas y cuestiones con o sin la supervisión y evaluación del profesor). Concretamente, se van a comparar los datos de los cursos académicos 2015-2016, 2016-2017 y 2017-2018.

Las actividades planificadas en la asignatura con fines de aprendizaje y evaluación han ido cambiando con el tiempo. En esencia, han aumentado las actividades realizadas por los estudiantes a través de la plataforma Moodle, con el fin de tener una corrección automática con feedback más rápido para los alumnos. El siguiente cuadro resume el trabajo del estudiante en la asignatura, tanto en lo que se refiere las sesiones presenciales de prácticas como de clases magistrales, indicándose en rojo las actividades con peso en la calificación final.

Tabla 1. Actividades planificadas dentro y fuera del aula

\begin{tabular}{|c|c|c|}
\hline & Sesiones prácticas & Clases \\
\hline Trabajo previo & $\begin{array}{l}\text { - Estudio de guion y apuntes } \\
\text { - Visualización de videos } \\
\text { - Cuestionario previo }\end{array}$ & $\begin{array}{ll}\text { - } & \text { Estudio con textos } \\
\text { - } & \text { Visualización de videos } \\
\text { - } & \text { Cuestionario tras cada video }\end{array}$ \\
\hline $\begin{array}{l}\text { Trabajo } \\
\text { presencial }\end{array}$ & $\begin{array}{l}\text { - Resumen del profesor } \\
\text { - Resolución de dudas } \\
\text { - Resol. de problemas complejos } \\
\text { - Aprendizaje del uso de software } \\
\text { - Entrega de resultados }\end{array}$ & $\begin{array}{l}\text { - Explicación del profesor } \\
\text { - } \text { Resolución de dudas } \\
\text { - Aprendizaje basado en problemas } \\
\text { - Gamificación con Kahoot! }\end{array}$ \\
\hline $\begin{array}{l}\text { Trabajo } \\
\text { posterior }\end{array}$ & & $\begin{array}{l}\text { - } \text { Cuestionarios de auto-evaluación } \\
\text { - Resol. problemas complejos } \\
\text { - Entrega de los resultados } \\
\text { - Resolución de cuestionarios }\end{array}$ \\
\hline
\end{tabular}

(c)) EY-NG-ND 2018, Universitat Politècnica de València

Congreso In-Red (2018) 
La metodología seguida en las clases se enmarca bajo el modelo de clase inversa en gran medida y se encuentra más detallada en las referencias (Peña, 2017a-b). Esencialmente, para las clases prácticas, se proporcionan una serie de materiales (guion, apuntes, videos) con los que el estudiante prepara la práctica y resuelve un cuestionario que en los cursos 2015-2017 se entregaba en papel al comienzo de la práctica y el presente curso se realiza a través de la plataforma Moodle. En la sesión, el profesor realiza un breve resumen, resuelve las dudas y deja el resto del tiempo para que los estudiantes por parejas reuelvan un problema más complejo. Si han trabajado adecuadamente, entregan los resultados al final de la sesión y no tienen que realizar tareas adicionales.

Con respecto a las sesiones magistrales, el profesor proporciona apuntes, bibliografía y videos con el fin de que el alumno prepare las clases (Zabalza, 2016-2017). Para el presente curso académico, tras cada video el alumno puede afianzar los conceptos más importantes mediante la resolución de un cuestionario breve que le sirve como autoevaluación. Una vez en clase, dependiendo del tema, el profesor explica en mayor o menor medida los conceptos y procedimientos teóricos para luego resolver problemas, bien el profesor, bien los alumnos en pequeños grupos bajo la guía y supervisión del profesor. Al finalizar cada tema, se realiza una gamificación con Kahoot!, cuyos resultados quedan fuera del alcance del presente artículo (Peña, 2018). También se proporciona al estudiante un cuestionario en Moodle con 3 intentos (contando en la nota sólo el último) y un trabajo que consiste en resolver varios problemas similares a los del examen, cuyos resultados debe entregar tanto en papel como a través de la plataforma Moodle para una corrección automatizada. Tras revisar la versión en papel se mantiene o se modifica la calificación obtenida de la corrección automática del trabajo.

Puesto que una buena parte de la evaluación y bastantes actividades de aprendizaje se realizan a través de Moodle, se dispone de una gran cantidad de información almacenada en la plataforma que puede servir para hacer seguimiento del proceso de enseñanzaaprendizaje. En la sección siguiente se presentan y comparan los datos registrados durante los 3 últimos cursos académicos, pero antes de eso, se detallan de forma concreta las diferencias en las actividades y recursos disponibles en la plataforma digital con el fin de que se pueda realizar una correcta interpretación de los resultados .

\section{Diferencias y novedades en cada curso analizado}

- El número de alumnos matriculados en cada curso ha sido 51 en 2015-2016, 70 en 2016-2017 y 52 en el curso actual.

- Las fechas del examen de la convocatoria de febrero, que pueden interesar a efectos de la actividad temporal en Moodle, fueron: 27/01/16, 02/02/17, 05/02/2018.

- Encuestas: durante los cursos 2015-2016 y 2016-2017 se realizaron encuestas sobre metodología y sobre su opinión acerca de los videos docentes, cuyos resultados se encuentran detallados en (Zabalza, 2016) y en (Zabalza, 2017).

- Cuestionarios de evaluación y de autoevaluación: han ido aumentando en número con el tiempo (5 en el curso 2015-2016, 7 en el curso 2016-2017 y 8 en el curso actual).

(cc) EY-NC-ND 2018, Universitat Politècnica de València

Congreso IN-RED (2018) 
- Videos docentes: los videos de la lección 2 mencionada a lo largo del documento se desarrollaron entre diciembre y julio de 2016, por lo que no estuvieron disponibles durante la impartición de esa lección (en octubre) y sólo los dos primeros estuvieron disponibles para la convocatoria de febrero.

- Clase inversa: innovación introducida en el curso 2016-2017.

- Cuestionarios tras cada video: innovación introducida en el curso 2017-2018.

- Formularios para introducir los resultados de los trabajos tutorados: innovación introducida en el curso 2017-2018.

\section{Resultados}

La plataforma digital utilizada en la Universidad de Zaragoza es Moodle, concretamente, es la versión 3 desde el curso 2016-2017 (curso 16-17 Moodle 3.1 y curso 17-18 Moodle 3.3). Hasta la fecha no se ha instalado ningún módulo para el análisis del aprendizaje, del tipo SmartKlass o Calam, por lo que en este trabajo se ha extraído directamente la información de cada curso y se ha analizado en hojas de cálculo. De la información almacenada en el curso de la asignatura, se presentan concretamente el análisis y la comparación de los siguientes datos:

- Actividad global del estudiante: número de vistas totales y número de mensajes intercambiados.

- Visualización de los videos y acceso a los cuestionarios correspondientes.

- Actividad asociada a los cuestionarios de evaluación: tiempo invertido, número de intentos, calificación.

- Uso de los cuestionarios de autoevaluación y de otros recursos colgados en Moodle.

\subsection{Actividad global en la plataforma}

La información sobre la actividad global puede encontrarse en el menú Administración, Informes, Estadísticas. Se contabilizan tanto el número de Vistas (“views”) de cualquier elemento del curso, como lo que Moodle denomina Mensajes ("posts”) (Moodle, 2013). Las Vistas incluyen actividades pasivas del usuario, como el acceso o visualización de recursos, lecturas o descargas. Los Mensajes engloban respuestas activas del usuario, como participación en foros, envío de tareas, resolución de cuestionarios o encuestas.

En las gráficas de la Figura 1 se muestran los resultados de los tres últimos cursos a lo largo del semestre (septiembre-febrero). Se observa un patrón de uso completamente diferente. Durante el curso 2015-2016, la actividad en general fue mucho más baja que en lo dos cursos posteriores, del orden de la mitad de accesos totales, mostrando un pico durante el mes de febrero, asociado a la proximidad del examen final. Esto puede deberse a un menor número de tareas encomendadas a través de la plataforma. 

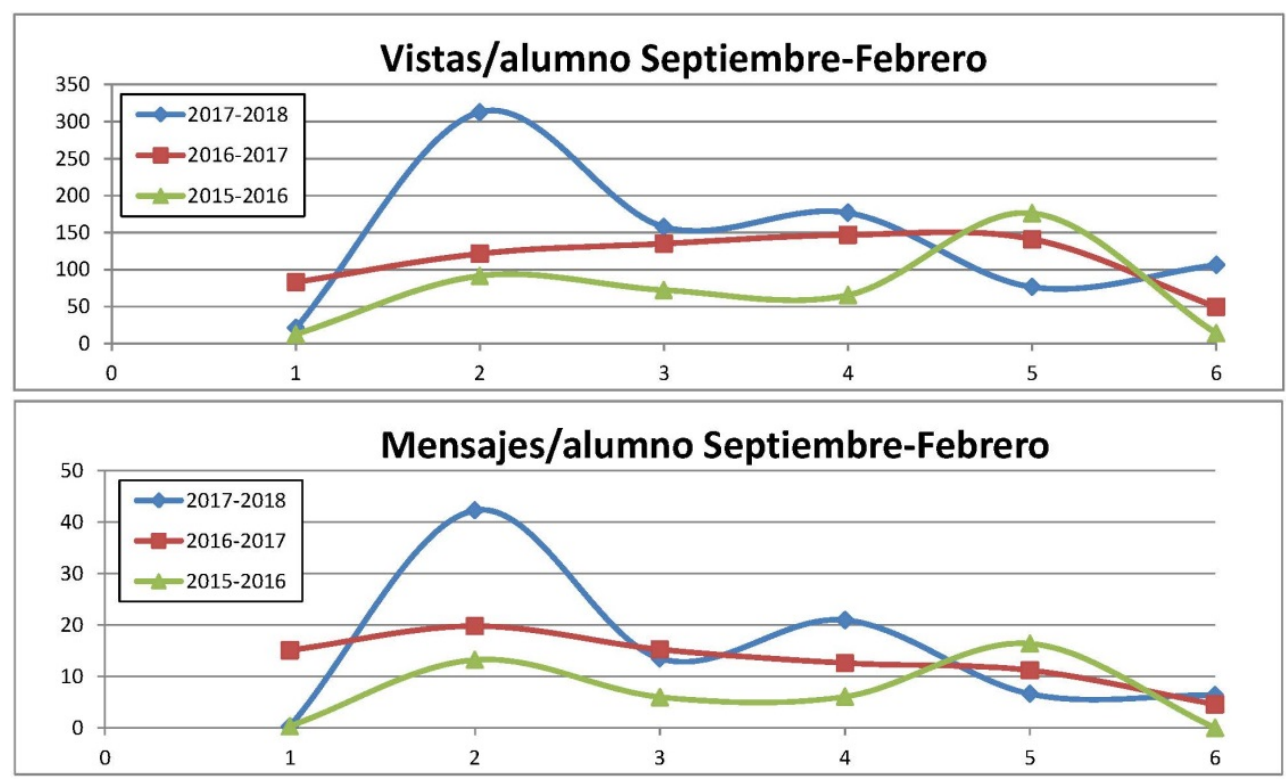

Fig. 1 Actividad general por alumno registrada en el curso de Moodle.

Entre los cursos 2016-2017 y 2017-2018 también existen diferencias significativas. El perfil de accesos durante el curso 2016-2017 es muy plano, mantiendo el nivel hasta el mes de enero en que se realizó el examen. Por el contrario, en el curso 2017-2018 observa una gran actividad durante el mes de octubre para luego tomar una tendencia decreciente con un repunte en el mes de diciembre. Llama la atención la baja actividad durante los meses de enero y febrero en que está la banda de preparación y realización del examen final.

\subsection{Visualización de videos docentes}

Un total de 20 videos docentes se están utilizando actualmente en la asignatura: 7 de ellos para la preparar las sesiones de prácticas, 6 para preparar las clases de la lección 2 que se ha impartido como clase inversa (Flipped Classroom Model, FCM) durante los cursos 20162017 y 2017-2018, y 7 videos más disponibles en el canal de YouTube de la Universidad Politécnica de Valencia. Los resultados obtenidos de las encuestas de opinión realizadas a los alumnos fueron muy positivos y se analizaron con detalle en publicaciones previas (Zabalza, 2016-2017).

Como ya se ha explicado en la Sección 2, no todos ellos han estado disponibles para todos los cursos académicos, hecho que se refleja en las gráficas de la Figura 2. Se ha representado el número de visualizaciones totales, el número de estudiantes que han visto al menos una vez el video y el porcentaje que ese número representa frente al número de matriculados en la asignatura. Nótese la diferencia de escala en el eje de abscisas: [0, 140], $[0,450]$ y $[0,300]$. 


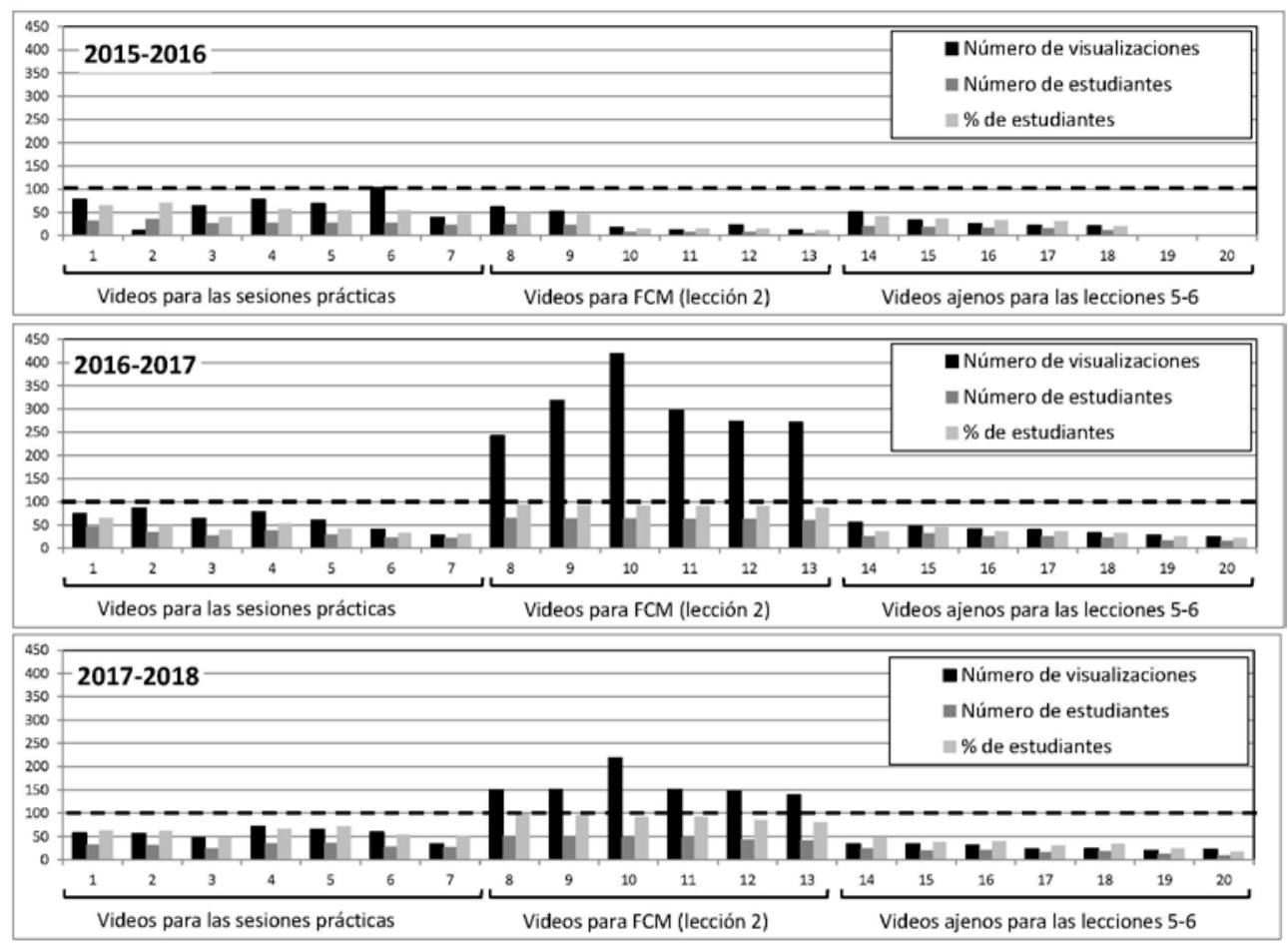

Fig. 2 Uso de los videos docentes.

Por ser de una escala mucho menor, no se ha representado el número de visualizaciones por alumno en la figura anterior. Los datos para cada bloque de videos (B1-prácticas, B2-FCM y B3-ajenos) se recogen en la Tabla 2:

Tabla 2. Resumen sobre la visualización de videos docentes

\begin{tabular}{ccccccc}
\hline \multirow{2}{*}{ Curso } & \multicolumn{3}{c}{ Estudiantes (\%) } & \multicolumn{3}{c}{ Vistas/alumno } \\
& B1 & B2 & B3 & B1 & B2 & B3 \\
\hline $2015-2016$ & 55,54 & 25,49 & 23,53 & 1,24 & 1,10 & 0,60 \\
$2016-2017$ & 45,20 & 91,67 & 34,08 & 0,89 & 4,34 & 0,56 \\
$2017-2018$ & 59,62 & 90,71 & 33,24 & 1,07 & 3,07 & 0,52 \\
\hline
\end{tabular}

Durante el primer curso, los videos más vistos fueron los destinados a prácticas. Por el contrario para los dos últimos cursos, aunque se mantienen niveles parecidos en el bloque B1, los videos más vistos fueron los de la lección 2. El porcentaje de alumnos que han visto los videos del bloque B2 es similar en los cursos 2016-2017 y 2017-2018, pero el número de visualizaciones ha disminuido en un $30 \%$ en el último curso, a pesar de tener asociado un cuestionario para ser respondido a continuación. 
Los videos del tercer bloque han mantenido cifras muy similares durante los tres cursos analizados. Aunque son videos de gran calidad y el profesor los considera de gran interés para los temas finales, los alumnos hacen un bajo uso de los mismos. El hecho de que estén desarrollados por un profesor externo a la Universidad de Zaragoza parece dar la impresión de que son complementarios, no esenciales para la asignatura.

Para el presente curso se han desarrollado cuestionarios específicos que le sirvan a estudiante para saber si ha aprendido lo más importante de cada video. Para este primer año, hay un cuestionario por cada dos videos de contenido relacionado y su resolución es requisito imprescindible para acceder a los videos siguientes. En la siguiente tabla se resume la actividad recogida a este respecto. La notación utilizada es la siguiente: Estudiantes, I-ntentos, T-iempo.

Tabla 3. Resumen sobre los cuestionarios asociados a cada video para el curso 2017-2018

\begin{tabular}{ccccc}
\hline Curso & E (\%) & I/E & T/I (min) & Nota media \\
\hline Videos 1-2 & 96,2 & 1,2 & 9 & 8,3 \\
Videos 3-4 & 88,5 & 1,1 & 10 & 9.2 \\
Videos 5-6 & 76,9 & 1,1 & 9 & 9,8 \\
\hline
\end{tabular}

A priori, un elevado porcentaje de alumnos ha completado al menos una vez el cuestionario, obteniendo una nota elevada. Dado que no había videos posteriores, no existía ninguna medida coercitiva para la realización del último cuestionario, reduciéndose el porcentaje de estudiantes que accedieron a él.

\subsection{Cuestionarios de evaluación}

Los cuestionarios de evaluación consisten en un conjunto de preguntas de diversos tipos (verdadero/falso, elección múltiple, calculadas) cuya nota promedio constituye un 5\% de la nota final. En los tres cursos analizados, el alumno dispone de 3 intentos contándose únicamente la nota del último. Durante los dos primeros cursos analizados, tras cada intento el alumno podía ver la calificación final pero no la puntuación de cada pregunta. A petición de los estudiantes esto se modificó para el presente curso, pudiéndose ver ahora si una respuesta es o no correcta tras completar cada intento. El número de preguntas y cuestionarios se ha ampliado con el tiempo, de ahí que con fines comparativos se haya calculado el tiempo dedicado por pregunta. 
Tabla 4. Resumen sobre los cuestionarios de evaluación

\begin{tabular}{cccccc}
\hline Curso & E (\%) & I/E & T/I (min) & T/P (min) & Nota media \\
\hline $2015-2016$ & 71,4 & 1,7 & 24 & 2,4 & 5,3 \\
$2016-2017$ & 73,3 & 1,7 & 22 & 1,9 & 6,6 \\
$2017-2018$ & 77,4 & 1,8 & 15 & 1,0 & 7,3 \\
\hline
\end{tabular}

En la Tabla 4 se ha utilizado la siguiente notación: E-studiantes, I-ntentos, T-iempo, Pregunta. Puede verse que la participación en esta actividad en cuanto a la proporción de estudiantes y al número de intentos es similar en todos los cursos. Sin embargo, el tiempo dedicado a su resolución ha disminuido con el tiempo, a la vez que ha aumentado considerablemente la nota media. Esto puede deberse en cierta medida a que ahora pueden saber si una respuesta es correcta o no tras cada intento. Aunque en ciertas preguntas hay una pequeña penalización por respuesta incorrecta, no se aplica a todas ellas y puede contribuir a conseguir una calificación más alta.

\subsection{Cuestionarios de autoevaluación y otros recursos de feedback}

Los cuestionarios de evaluación se convierten en herramientas de autoevaluación para que el alumno los realice tantas veces como considere oportuno. Siguiendo la notación de tablas anteriores, en la Tabla 5 se recoge el número total de intentos, el porcentaje de estudiantes que ha realizado al menos un intento y el promedio de intentos por alumno.

Tabla 5. Resumen sobre los cuestionarios de autoevaluación

\begin{tabular}{cccc}
\hline Curso & I & E (\%) & I/E \\
\hline $2015-2016$ & 303 & 29,9 & 2,5 \\
$2016-2017$ & 285 & 35,3 & 1,6 \\
$2017-2018$ & 140 & 25,2 & 1,3 \\
\hline
\end{tabular}

El número de intentos ha ido disminuyendo con el tiempo, así como parece estar disminuyendo también el porcentaje de alumnos que los resuelve. Esto puede deberse a fluctuaciones naturales entre grupos de estudiantes diferentes, pero también puede ser que el aumento en las actividades obligatorias en la asignatura reduzca el interés por recursos de uso no obligatorio.

A partir del curso 2016-2017, junto con la calificación de cada trabajo tutorado se cuelga un resumen de los errores más comunes/importantes detectados en las diferentes entregas. A pesar de los recordatorios enviados por el profesor, estos documentos han tenido una baja acogida por parte de los estudiantes. Tan sólo un $27 \%$ de los estudiantes lo consultó en el curso 2016-2017 y un 18\% en el curso actual.

(c)) EY-NC-ND 2018, Universitat Politècnica de València

Congreso In-Red (2018) 


\subsection{Comparativa de resultados}

Una vez presentada la información obtenida de la plataforma Moodle, se quiere discutir en este apartado final su posible uso para correlacionar o predecir con dicha información los resultados en el examen final. En la siguiente tabla se recoge el número de estudiantes matriculados en cada curso, las calificaciones promedio en diferentes actividades y el porcentaje de estudiantes aprobados en la primera convocatoria.

Tabla 6. Comparación de resultados de aprendizaje

\begin{tabular}{lccc}
\hline Curso & 2015-2016 & 2016-2017 & 2017-2018 \\
\hline Número de alumnos matriculados & 42 & 69 & 52 \\
Nota en cuestionarios de Moodle & 5,3 & 6,6 & 7,3 \\
Nota de trabajos tutorados & 6,8 & 7,4 & 7,7 \\
Nota media del examen final & 6,5 & 6,2 & 6,4 \\
Estudiantes presentados (\%) & 90,7 & 91,3 & 90,4 \\
Estudiantes aprobados (\%) & 48,7 & 66,7 & 45,8 \\
\hline
\end{tabular}

La nota media de los estudiantes aprobados es muy parecida durante los tres cursos académicos, así como el porcentaje de alumnos presentados, que es muy elevado. Las calificaciones en los cuestionarios de Moodle y en los trabajos tutorados ha ido aumentando en cada curso, pero no ha ocurrido lo mismo con el porcentaje de éxito en el examen final. En el curso 2016-2017 la tasa de aprobados supera en prácticamente 20 puntos porcentuales a los otros dos grupos.

\section{Conclusiones}

Revisando los datos presentados sobre la actividad en Moodle, se puede relacionar la tasa de éxito con ciertos indicadores de buen uso de los recursos recomendados por el profesor.

- Para el curso 2016-2017 se observa un uso continuado y estable, sin picos al comienzo o al final del cuatrimestre.

- El número de visualizaciones de los vídeos de la lección 2 es más alto durante este curso, a pesar de que dicho año no se encontraban disponibles los cuestionarios para afianzar lo aprendido con cada video.

- Con respecto a los cuestionarios de evaluación, el tiempo dedicado a cada pregunta se ha reducido desde $2.4 \mathrm{~min}$ hasta $1.0 \mathrm{~min}$, pero la nota media ha subido 2 puntos. Teniendo en cuenta los resultados del examen, podría decirse que han existido malas prácticas durante la realización de los mismos. Igual que se han detectado muchos más casos de copia fraudulenta en los trabajos tutorados durante el presente curso, podría pensarse que ha ocurrido lo mismo en los cuestionarios de evaluación de la parte teórica.

(cc) EY-NC-ND 2018, Universitat Politècnica de València

Congreso IN-RED (2018) 
- En cuanto a los cuestionarios de autoevaluación, el porcentaje de estudiantes que realizó al menos un intento es superior en 10 puntos porcentuales durante el curso 2016-2017, aunque el número de intentos por estudiante es menor que en el curso previo.

- También la consulta de los errores frecuentes en los trabajos tutorados fue superior durante el curso 2016-2017 en 10 puntos porcentuales con respecto al curso actual.

Hay que añadir que existen factores externos a la asignatura que influyen notablemente en la dedicación del estudiante y que pueden variar de un curso a otro, pero que no son completamente conocidos por el profesor y de ninguna manera controlables por él. La existencia de exámenes intermedios y de entrega de trabajos exigentes hace que descienda notablemente la asistencia a clase y el seguimiento continuo de la asignatura.

De todo esto, se pueden obtener varias conclusiones:

- Conviene reducir las posibilidades de copia en los cuestionarios de evaluación, reduciendo por ejemplo el número de intentos, eliminando la opción de saber si una pregunta es correcta, limitando el tiempo para su resolución o seleccionando preguntas de forma aleatoria de un banco de preguntas.

- Para un mejor aprovechamiento de las sesiones de prácticas, será útil realizar una breve evaluación (diferente para cada grupo de prácticas) al comienzo de la sesión, pudiendo realizar la práctica únicamente los alumnos que realmente la han preparado.

- Sobre los trabajos tutorados, el uso de formularios para introducir los resultados durante este curso para automatizar la corrección, parece haber fomentado la copia fraudulenta, ya que se han detectado bastantes más casos que en el curso anterior. Convendrá tomar medidas coercitivas o diseñar casos múltiples (aunque es complicado implementar preguntas múltiples calculadas en Moodle) para reducirlo, ya que este comportamiento sólo le proporciona al alumno una falsa sensación de llevar bien la asignatura y reduce su tasa de éxito en el examen final.

- Aunque realmente no es el cometido del profesor, parece necesario recordar al estudiante la utilidad de recursos como videos, cuestionarios de autoevaluación o resúmenes para realizar un aprendizaje significativo y eficaz.

- Implementar una evaluación por rúbricas para que el estudiante tenga claros sus avances en el curso, puede ser motivador pero hay que encontrar una forma eficiente de gestionarlas, ya que la herramienta de Moodle no es una opción asumible en grupos tan grandes como los que existen en este grado (Peña, 2018).

Para finalizar, cabe señalar que el estudio resultaría más eficaz a efectos de predicción de la tasa de éxito si el análisis de los datos se hubiera realizado por estudiante, en lugar de utilizar valores promedio. Sin embargo, esta tarea es muy costosa si no se dispone de herramientas que automaticen el análisis. En este sentido, resultará útil disponer en próximos cursos de la nueva versión Moodle 3.4 que incorpora un módulo de analíticas del aprendizaje (Moodle, 2018). 
Uso de recursos y rendimiento en las actividades evaluación: análisis y comparación de resultados en el Grado en Ingeniería de Tecnologías Industriales

\section{Referencias}

BOYER, A. y BONNIN, G. (2016) Higher Education and the Revolution of Learning Analytics. Report of the International Council for Open and Distance Education (ICDE). Disponible en: $<$ https://icde.memberclicks.net/assets/RESOURCES/anne_la_report\%20cc\%20licence.pdf> [Consulta: 15 de marzo de 2018].

FERGUSON, R., et al. (2016). Research Evidence on the Use of Learning Analytics - Implications for Education Policy. R. Vuorikari, J. Castaño Muñoz (Eds.). Joint Research Centre Science for Policy Report; EUR 28294 EN; doi:10.2791/955210.

MOODLE (2013). Course overview report. Documentación de Moodle.org. $<$ https://docs.moodle.org/25/en/Course_overview_report > [Consulta: 9 de marzo de 2018].

MOODLE (2018). Analytics. Documentación de Moodle.org. $<$ https://docs.moodle.org/34/en/Analytics> [Consulta : 15 de mayo de 2018].

PEÑA, B. et al. (2017a) "Pilot experience for the application of the flipped classroom in subjects of the field of Thermal Engineering”. En: INTED17 Proceedings of the $11^{\text {th }}$ annual International Technology, Education and Development Conference, Valencia, IATED Academy, pp. 3601-3610. Doi: 10.21125/inted.2017.0887.

PEÑA, B. et al. (2017b) “Experiencia piloto de aula invertida para mejorar el proceso de enseñanzaaprendizaje en la asignatura de Termodinámica Técnica”. En: Actas del congreso INRED 2017, Valencia, Editorial Universitat Politècnica de València. Doi: 10.4995/INRED2017.2017.6868. Disponible en: < http://ocs.editorial.upv.es/index.php/INRED/INRED2016/paper/view/4430>.

PEÑA, B. (2018) “Defining quantitative and automated rubrics from assessment activities scores” En: INTED18 Proceedings of the $12^{\text {th }}$ annual International Technology, Education and Development Conference, Valencia, IATED Academy, pp. Doi:

SCLATER N., PEASGOOD A. y MULLAN J. (2016) "Learning Analytics in Higher Education: A review of UK and international practice” Full Report: JISC. Disponible en: $<$ https://www.jisc.ac.uk/reports/learning-analytics-in-higher-education> [Consulta:11 de marzo de 2018].

ZABALZA, I., PEÑA, B., LLERA, E.M. y USÓN, S. (2016) "Improving the teaching-learning process using educational videos as reusable learning objects in the field of thermal engineering”, Proceedings of the $8^{\text {th }}$ International Conference on Education and New Learning Technologies (EDULEARN 2016), Barcelona, IATED Academy, pp. 363-372. Doi: 10.21125/edulearn.2016.1068.

ZABALZA, I., et al. (2017) "Development of educational videos as reusable learning objects for their integration into an Open Courseware on fundamentals of thermodynamics and thermal engineering”, En: INTED17 Proceedings of the $11^{\text {th }}$ annual International Technology, Education and Development Conference, Valencia, IATED Academy, pp. 4453-4461. Doi: 10.21125/inted.2017.1055.

WANLI X., RUI G., EVA P. y SEAN G. (2015) "Participation-based student final performance prediction model through interpretable Genetic Programming: Integrating learning analytics, educational data mining and theory" Computers in Human Behavior 47 (2015) 168-181.

(cc) EY-NC-ND 2018, Universitat Politècnica de València

Congreso IN-RED (2018) 